«Si I'on rate le premier bouton, on ne peut pas boutonner correctement sa chemise»: c'est ainsi qu'un manuel de jurisprudence cite Goethe.

Dans I'histoire qui suit, on a manifestement raté ce premier bouton et continué à boutonner comme si de rien n'était. A la lecture du jugement, qui se conclut par un acquittement, on peut à juste titre se poser la question suivante: combien de diligence et de pondération l'Etat attend-il d'un institut de médecine légale qui influence de manière décisive le fait qu'un médecin pénitentiaire lequel travaille dans des conditions extrêmement difficiles - soit accusé ou non d'homicide par négligence? Ce procès a duré plus d'une année et englouti des fonds publics sous forme de ressources financières et humaines. Et si l'on peut imaginer la pression subie par l'accusé, il n'est pas possible de la quantifier. II reste à espérer que cette procédure n'aura pas fait que causer coûts et colère, mais qu'on en tirera aussi les leçons qui s'imposent.

En outre, dans une telle situation, on devrait au moins pouvoir attendre de l'Etat qu'en sa qualité de mandant ou d'employeur, il accorde sans autres une assistance juridique à ses médecins pénitentiaires. Dans le cas contraire, on ne saurait que recommander aux médecins de refuser d'assumer des tâches aussi ingrates.

Lucia Rabia, service juridique de la FMH

\title{
Gefängnisarzt - mit beiden Beinen im Gefängnis?
}

Reto Gross, Andreas Würmli

Korrespondenz:

Dr. med. Reto Gross

Facharzt für Allgemeinmedizin FMH

Bahnhofstrasse 21a

CH-9450 Altstätten

Tel. 0717556377

Fax 0717556360

rgross@hin.ch

Dr. med. Andreas Würmli

Facharzt für Kinder- und

Jugendmedizin FMH

Trogenerstrasse 20

CH-9450 Altstätten

Tel. 0717553577

Fax 0717553578

praxis.wuermli@bluewin.ch
Der Todesfall eines Häftlings im Januar 2006 hat ein weites Medienecho gefunden. Der Fall ist inzwischen endgültig abgeschlossen. Zwei niedergelassene, angeschuldigte Ärzte nehmen dazu Stellung. Der eine wurde als stellvertretender Gefängnisarzt und der andere als Notfallarzt in das Verfahren hineingezogen. Im ersten Teil schildern sie nochmals kurz die Fakten, um dann im zweiten Teil ihre Bedenken über den Verlauf des Verfahrens darzulegen. Als Kernaussage kommen sie zu folgendem Schluss: Unser Justizsystem ist selbst mit einfachen medizinischen Fakten überfordert. Daraus resultiert eine grosse Abhängigkeit von medizinischen Gutachten. Deshalb sollte in Zukunft bei solchen Gutachten mindestens das Peer-review-Prinzip als Voraussetzung gelten, und der Gutachter selbst darf nur in seinem ganz spezifischen Fachgebiet Auskunft geben.

\section{Sachverhalt, dargestellt durch den stellvertretenden Gefängnisarzt}

Ein 21jähriger Mann aus Guinea wird wegen Drogenhandels verurteilt. Nach der Verurteilung entwickelt er ein auffälliges Verhalten, was wenige Tage vor Weihnachten 2005 die Verlegung in ein anderes Gefängnis zur Folge hat. Der Häftling verhält sich aggressiv und zerstört einen Teil des Zellenmobiliars, dann sitzt er nackt in der Zelle und verweigert das Essen, er wäscht sich mit dem Putzwasser den Kopf bzw. versucht dieses zu trinken. Dieses auffällige Verhalten wird von mir als stellvertretender Gefängnisarzt am 26. Dezem- ber 2005 als Protesthaltung im Sinne einer Pseudodemenz beurteilt, wie sie bei Gefangenen gelegentlich auftritt [1]. Die Auffälligkeit des Inhaftierten konzentriert sich in der Folge zunehmend auf eine demonstrative Nahrungs- und Trinkverweigerung, wobei allerdings durch die Betreuenden nicht objektivierbar ist, ob tatsächlich nichts getrunken wird (die Zelle verfügt über fliessendes Wasser, Tranksame werden ausgeleert). Ich empfehle eine abwartende, symptomatische Behandlung, insbesondere das Bereitstellen von genügend Trinkflüssigkeit und soweit möglich menschliche Zuwendung.

Am 1. Januar 2006 wird beim Gefangenen durch das Gefängnispersonal (medizinische Laien) mit dem Ohrthermometer eine sehr tiefe Körpertemperatur gemessen und daraufhin der regionale Notfallarzt gerufen. Dieser untersucht den Inhaftierten, der sich körperlich so wehrt, dass er die Blutdruckmessung verunmöglicht. Der Arzt beurteilt den Zustand als nicht lebensbedrohlich. Die Betreuer im Gefängnis ergreifen sinnvolle und effektive Massnahmen zur Sicherstellung der Köpertemperatur (Fussbodenheizung auf Maximalstufe, Decken, Kleider) und flössen dem Gefangenen warmen Tee ein, wogegen sich dieser nicht wehrt.

Am nächsten Tag, dem 2. Januar 2006, untersuche ich den Gefangenen erneut. Meine Beurteilung lautet: deutlich reduzierter Allgemeinzustand infolge von Nahrungs- und wahrscheinlich auch Trinkverweigerung, keine unmittelbare 
Lebensgefahr. Ich weise den Gefangenen jedoch darauf hin, dass sein Verhalten für ihn lebensgefährlich sein kann. Die Auffälligkeiten ergeben keine wesentlichen neuen Gesichtspunkte. Die Gefangenenbetreuung ist durch den pflegerischen Aufwand aber an der Grenze ihrer Möglichkeiten. Ich empfehle für den folgenden Tag eine Hospitalisation in einem Gefängnisspital. Zum Zeitpunkt der Untersuchung am 2. Januar 2006 - rückblickend wenige Stunden vor dem Tod des Häftlings - ergeben sich für mich keine Hinweise auf das Vorliegen einer akut lebensbedrohlichen Situation.

In der darauffolgenden Nacht stirbt der Gefangene. Ein gerichtsmedizinisches Gutachten nennt als wesentliche Todesursache einen Flüssigkeitsmangel und erhebt schwere Vorwürfe gegen die beiden behandelnden Ärzte. Der Flüssigkeitsmangel wird durch eine Art Hämatokrituntersuchung im Leichenblut und die Restwasserbestimmung in der Muskulatur «objektiviert». Nicht schlüssig erklärt wird jedoch der Befund in der Blase: $500 \mathrm{ml}$ Urin mit normalem spezifischem Gewicht. Eine Schätzung des wahrscheinlichen Todeszeitpunktes wird ebenfalls nicht vorgenommen [2]. Das rechtsmedizinische Gutachten ist Grundlage für die Eröffnung einer Strafuntersuchung. Die darin genannte Todesursache wird öffentlich gemacht, und der angebliche Tod durch Verdursten in den folgenden Wochen von den nationalen Medien breitgeschlagen. Aus den objektiven Obduktionsbefunden lässt sich diese Todesursache für uns als angeschuldigte Ärzte allerdings nicht nachvollziehen. Der Häftling verstarb mit grösster Wahrscheinlichkeit an den Folgen einer akuten Lungenembolie, die in der gerichtsmedizinischen Sektion ebenfalls festgestellt worden ist. Wir verlangen ein zweites Gutachten, werden aber noch vor einer zweiten Fachmeinung vom Untersuchungsrichter zu einer Einvernahme vorgeladen und fahren mit unseren Anwälten über 90 Kilometer quer durch den Kanton (und zurück) zur Einvernahme, wo wir die Aussage verweigern.

Schliesslich wird doch noch ein zweites Gutachten eingeholt. Es stammt von einem 74jährigen pensionierten internistischen Spitalchefarzt, der selbst keine Erfahrung als Gefängnisarzt hat. Der Gutachter behauptet, der stellvertretende Gefängnisarzt hätte den Patienten ungenügend untersucht, ohne jedoch zu sagen, was bei der Untersuchung unterlassen wurde.

11 Monate nach dem Tod des Häftlings werde ich als stellvertretender Gefängnisarzt aufgrund des zweiten Gutachtens der fahrlässigen Tötung angeklagt. Diese Tatsache erfahre ich zuerst von meinem jüngsten, 14jährigen Sohn, der es in den Mittagsnachrichten von Radio
DRS 3 hört. Der entsprechende Brief der Staatsanwaltschaft erreicht meinen Anwalt erst einige Tage später.

14 Monate nach dem Geschehen findet die Verhandlung in erster Instanz vor dem Kreisgericht statt. Sie endet mit einem vollumfänglichen Freispruch für mich. Das Urteil ist inzwischen rechtskräftig.

\section{Bezüglich menschlicher, rechtlicher und fachlicher Unterstützung ist anzufügen}

Die Anklagekammer stellt bei ihrem Entscheid fest, dass die beiden Ärzte in dieser Sache in Beamtenfunktion gehandelt hätten. Der Kanton lehnt unser Gesuch um Rechtshilfe aber mehrfach ab. Erst die konkrete Zustellung des kantonalen Personalreglements an die zuständige Regierungsrätin ändert diese Haltung des Kantons und uns Ärzten wird Rechtsbeistand bis zur ersten Instanz gewährt.

Mehrere Rückfragen bei verschiedenen schweizerischen Rechtmedizinern - auch bei ehemaligen Studienkollegen - ergeben, dass es für die beiden angeschuldigte Ärzte in der Schweiz keine referenzierbaren Auskünfte aus diesem Fachgebiet geben wird. Nicht einmal banale Normwerte können genannt werden, die jeder selbst nachschlagen könnte (wenn er nur wüsste, wo!). Einzig der Gefängnisleiter unterstützt uns aktiv mit aktueller nichtmedizinischer Fachliteratur. Die kantonalen Behörden bzw. die Verwaltung (Gesundheits- und Justizdepartement) lassen in den 14 Monaten des Verfahrens nichts von sich hören. Fachlich sind wir beiden Ärzte in erster Linie auf uns selbst gestellt. Die regionalen Kollegen und die kantonale Ärztegesellschaft sichern uns ihre Unterstützung zu, was spürbaren Halt gibt.

Die Aufarbeitung der sachlichen und fachlichen Rechtfertigung des ärztlichen Tuns und Lassens während der wenigen entscheidenden Stunden der konkreten Situation beansprucht praktisch die ganze Freizeit während eines Jahres und belastet uns zwei Ärzte (und unser Umfeld!) schwer. Sie ist nur durch gute Englisch- und Internetkenntnisse überhaupt möglich (schliesslich referenzieren wir über 200 Internetweblinks). Dank zweier aufmerksamer und kompetenter Anwälte lassen sich die komplexen medizinischen/naturwissenschaftlichen Fakten in eine für Laien verständliche juristische Sprache umformulieren.

\section{Persönliches Fazit} als stellvertretender Gefängnisarzt

Die öffentlich bestellten Gutachter haben mehr zur Verwirrung als zur Lösung des Falls beigetragen, angesichts der unkritischen Expertenhörig- 
keit der Justizorgane gegenüber medizinischen Fachgutachten eine erschreckende Wahrnehmung. Daher ist zu fordern, dass medizinische Gutachten vor Gericht zumindest einen Peer-review-Prozess (Vier-Augen-Prinzip) durchlaufen.

Zur Strategie der Aussageverweigerung: «Reden ist Silber - Schweigen ist Gold». Die Vorstellung, die Untersuchungsorgane bzw. der Staatsanwalt seien tatsächlich nur an der Wahrheit interessiert, erwies sich als naive Staatskunderomantik. Meine Wahrnehmung war, dass sie vor allem Schuldige suchten. Ein Posthalter, eine Kindergärtnerin oder ein Jurist(!) wären an unserer Stelle in noch viel grössere Bedrängnis geraten.

Der Arzt (und wohl auch der gesunde Menschenverstand) ist seinerseits mit der Funktion der Justiz überfordert. Fazit: Besorgen Sie sich in solchen Nöten einen guten Anwalt!

\section{Persönliche Feststellungen zum Ereignis (vom Pädiater als Notfallarzt am 1. Januar 2006)}

Ein unangemeldetes Telefon erreicht mich in der vollen Sprechstunde: «Grüezi Herr Würmli, ich bin Staatsanwaltstellvertreter des Kantons und teile Ihnen mit, dass gegen Sie eine Strafuntersuchung eröffnet wird. Ich empfehle Ihnen dringend, einen Anwalt zu nehmen ...». Diese Mitteilung einer kantonalen Behörde war in kürzester Zeit erledigt - meine Welt damit auch! Seit kurzer Zeit praktizierend, wurde ich schon angeschuldigt. Ich war verunsichert, fühlte mich auf schwabbeligen Beinen, die Hände waren kaltschweissig - aber halt, ich bin ja noch in der Praxis und habe seriös weiterzufunktionieren. Schön lächeln und weiter geht's, nun leider noch mehr unter Zeitdruck, zum nächsten Patienten.

Zwei Monate (!) später erhalte ich die Akten, darunter auch das belastende gerichtsmedizinische Gutachten mit Autopsiebericht. Dieses wurde vor mir schon von mehreren Juristen sorgfältig (?) gelesen und anhand dessen wird die Strafuntersuchung gegen meinen Kollegen und mich eröffnet. Etwas erstaunt bin ich, in diesen von Fachpersonen durchgelesenen Berichten schon beim ersten Durchsehen einige offensichtliche Ungereimtheiten $\mathrm{zu}$ finden, die ich hier in aller Kürze aufzeige.

Ein scheinbar massiv auffälliger Hautturgor (Hautfalte post mortem verbleibt zwischen 3 und 4 Minuten) wird in der Autopsie nur bei der Hälfte der untersuchten Stellen am Integument gefunden, aber im Gutachten als fast beweisend für eine Exsiccose aufgeführt. Zur aspektmässigen Beschreibung des Wassergehaltes der Muskeln des Toten wird der Begriff «Trockenfleisch» verwendet (Wassergehalt von Trockenfleisch: um
$50 \%$. Gerichtsmedizinisch nachgewiesener Wassergehalt der Muskeln in der Autopsie: 72\%). Es wird im Gutachten ein Normwert von «rund 80\%» für den Wassergehalt der Muskulatur genannt, aber keine mögliche Streubreite diskutiert (Alter, Geschlecht, ethnische Zugehörigkeit, spezifischer Muskel). In der Literatur findet man bei in vivo entnommener Muskulatur auch Normwerte von 73\% [3]. Eine Lungenembolie auf der rechten Seite wechselt plötzlich auf die linke oder war sie doch rechts oder gar beidseits? Die Todesursache Unterkühlung wird zwar ausgeschlossen, aber trotzdem wird suggestiv erwähnt, dass eine gewisse Unterkühlung eine Rolle gespielt habe, da schon Patienten auf einem kalten Steinboden erfroren seien (tatsächlich aber war es ein Zellenboden mit funktionierender Bodenheizung). Eine volle Harnblase mit $500 \mathrm{ml}$ Urin mit einer normalen Osmolalität von $765 \mathrm{mOsm} / \mathrm{kg}$ wird nicht erklärt. Das Ohrthermometer wird vom Gerichtsmediziner mit einem selbst erfundenen Testverfahren geprüft, um mit scheinbarer Wissenschaftlichkeit dessen Funktionstüchtigkeit zu beweisen, ohne aber die Handhabungsprobleme bei medizinischen Laien (Temperaturmessung durch Gefängnispersonal) auch nur anzudeuten. Der Gerichtsmediziner erwähnt im Gutachten den GCS (Glasgow Coma Score). Ihm liegt mein schriftlicher Notfallbericht vor, wo im Status festgehalten ist: «Augen geöffnet, gezielte Abwehr, unverständliche Sprache, Patient nicht kooperativ ...» Daraus schliesst er im Gutachten, der GCS sei so tief gewesen, dass jeder Arzt, ja sogar nichtärztliche Medizinalpersonen, anders gehandelt hätten als wir. Beim zweiten Durchlesen findet man noch weitere Ungereimtheiten ... Praktisch all diesen Fragen geht die Staatsanwaltschaft trotz unseren ausführlichen erklärenden Hinweisen nie spezifisch nach!

Acht Monate nach dem ersten Gutachten datiert das von uns geforderte zweite Gutachten, verfasst von einem ehemaligen Chefarzt einer medizinischen Klinik (unsere Anregung war: entweder ein anderer Gefängnisarzt aus der Schweiz oder ein Gerichtsmediziner von ausserhalb der Schweiz). Der zweite Gutachter widerspricht dem ersten Gutachten völlig: Dehydrierung als Todesursache ist ausgeschlossen (deshalb wird das Verfahren - nach neun Monaten Zittern - gegen mich aufgehoben). Er anerkennt aber ausdrücklich die professionelle Qualität des ersten Gutachtens und lässt sich als Nichtpsychiater über psychiatrische Diagnosen aus. Eigentlich hätte er den Auftrag von der Staatsanwaltschaft gehabt, bei für ihn als Internisten nicht fachspezifischen Fragen einen geeigneten Fachspezialisten um sein schriftliches Urteil zu bitten. Entweder 
hat die Staatsanwaltschaft nicht bemerkt, dass der Zweitgutachter diesem Auftrag nicht nachgekommen ist, oder sie stört sich bewusst nicht daran.

Ein Untersuchungsrichter/stellvertretender Staatsanwalt darf sich erlauben, während der Einvernahme nicht auf meine Bitte einzugehen, mich kurz mit meinem Anwalt zu besprechen, was ein Grundrecht des Angeschuldigten wäre. Dieser einmalige Fauxpas hat keine Konsequenzen. Schliesslich ist es sogar möglich, dass der Staatsanwalt uns die Bitte abschlägt, ein Doppel des Einvernahmeprotokolls zu erhalten. Mein Anwalt darf aber alles von Hand abschreiben, bevor wir es unterzeichnen. Währenddessen müssen wir alle warten, selbst der Staatsanwalt!

\section{Konsequenz aus meinen Wahrnehmungen} Ich wünsche mir, dass Gerichtsmediziner, Fachgutachter, Untersuchungsrichter, Staatsanwälte und alle Beteiligten auch im Justiz- und Polizeiwesen sich so professionell und ethisch korrekt verhalten, wie sie es vom behandelnden Arzt verlangen. Der wirklich Professionelle kennt seine Schwächen und Grenzen. Das genaue Gegenteil habe ich in diesem Verfahren erlebt. Es hätte der gerichtlichen Untersuchung nur gedient, wenn der Untersuchungsrichter und die Gutachter bei gewissen Fragen ihr Defizit an exaktem Wissen geäussert hätten. Wenn der Untersuchungsrichter konkrete Fragen vor dem Erstellen des ersten Gutachtens an den Gerichtsmediziner stellt, müsste sich der Gutachter wahrscheinlich nicht an für ihn völlig fremde Themen wagen (Thermometer, GCS).

Es geht mir nicht um die Vergangenheit, sondern um die Zukunft. Um die Weichen für die Zukunft zu stellen, braucht man die Erfahrung der Vergangenheit.

Literatur

1 Butzke IF, Hofmann F, Offinger A, Stanga Z. Das Ganser-Syndrom: Scheinblödsinn oder Konversionsstörung? Schweiz Med Forum. 2005;5:299-300.

2 Mund MT, Bär W. Rechtsmedizinische Aspekte beim plötzlichen Todesfall. Schweiz Med Forum. 2005;5:129-35.

3 Flear CT, Carpenter RG, Florence I. Variability in the water, sodium, potassium and chloride content of human skeletal muscle. J Clin Path. 1965;18: 74-81.

\section{La médecine en milieu pénitentiaire: un exercice médical sous pression}

\section{Bruno Gravier}

Président de la Conférence des Médecins Pénitentiaires Suisses.

Correspondance: Prof Dr Bruno Gravier Service de médecine et de psychiatrie pénitentiaires Département de psychiatrie du CHUV Site de Céry CH-1008 Prilly
L'épisode dramatique relaté par nos confrères et ses suites judiciaires soulignent la difficulté de l'exercice médical en prison et sa particulière complexité. Les écrits de nos confrères illustrent aussi la difficulté du regard de l'expert amené à évaluer la pratique médicale dans un cadre aussi particulier et à déterminer s'il y a eu faute. Ils mettent aussi en lumière le rôle ingrat du juge lié aux conclusions que lui livrent les experts, et confronté à un domaine complexe, obscur et inconnu, et à des rapports écrits souvent de manière peu intelligible. On se rend compte combien la justice est peu armée, dans de tels cas, pour faire la part des choses entre respect de l'obligation de faire un usage judicieux des moyens à laquelle est tenu tout médecin ou erreur d'appréciation, voire faute médicale.

\section{Le médecin pénitentiaire en dilemme constant entre patient et administration} Le médecin en milieu pénitentiaire est régulièrement confronté à des situations cliniques ex- trêmes (grèves de la faim, suspicions de simulations, violences contre soi au contre autrui, etc.). Il se trouve aussi dans une situation de double loyauté qui le conduit à gérer des contradictions parfois insolubles: loyauté envers son patient à qui il doit les meilleurs soins possibles, loyauté envers l'administration pénitentiaire à qui il doit le respect des contraintes sécuritaires du lieu où il exerce.

Les directives de l'Académie Suisse des Sciences Médicales concernant l'exercice de la médecine pour les personnes détenues ont été publiées en 2002, après un épisode tout aussi dramatique, pour aider le médecin travaillant en milieu pénitentiaire et ses partenaires à disposer de points de repères clairs, en conformité avec le droit de la santé et avec les recommandations internationales. Ces directives rappellent les exigences fondamentales de la médecine en milieu pénitentiaire: confidentialité, indépendance médicale, refus d'associer le médecin aux aspects répressifs, principe d'équivalence avec les soins prodigués 
en milieu libre. Elles rappellent, en particulier, que la décision du gréviste de la faim doit être «médicalement respectée, même en cas de risque majeur pour la santé», à condition, bien sûr, que la capacité de discernement du patient lorsqu'il formule son refus d'alimentation soit entière. Ces principes cardinaux peuvent sembler évidents pour tout médecin qui n'a pas expérimenté la particularité de l'exercice médical en prison. Ceux qui y ont travaillé savent, en revanche, que la prison est un lieu où ces repères se perdent vite.

L'appréciation d'une urgence médicale en prison est toujours délicate et brouillée par des considérations qui dépassent le champ clinique et qui constituent autant de sources de pression et donc de biais.

La pathologie présentée par le patient est elle authentique ou majorée? Le patient simule-t-il pour obtenir un transfert en milieu hospitalier? L'hôpital est-il équipé pour accueillir des patients détenus? Ne reprochera-t-on pas au médecin une légèreté coupable dans l'appréciation de l'urgence au regard des exigences sécuritaires s'il s'empresse de déplacer le détenu à l'hôpital? Les tableaux cliniques sont souvent trompeurs: un patient qui refuse de s'alimenter est-il dans la revendication et la protestation? Présente-t-il, un trouble psychique altérant son discernement? Entre exacerbation d'un comportement et crise pathologique la marge est souvent mince. Dans ces situations d'urgence, le médecin ne connaît, bien souvent pas tous les enjeux et ne peut compléter son observation que sur la base des indications du personnel pénitentiaire, lui même peu à l'aise, de façon bien compréhensible, avec un tableau inquiétant.

Même délié du secret professionnel, que peutil dire des troubles présentés par son patient au personnel de surveillance, directeurs et gardiens, surtout lorsque le diagnostic est indécis? Par exemple, il est arrivé que l'existence de troubles fonctionnels ou de manifestations d'angoisse soit entendue par le personnel pénitentiaire comme une preuve de simulation et que celui-ci en tire argument pour sanctionner le détenu. Même en prison, la confidentialité de l'acte médical est primordiale.

Pourtant, le médecin est souvent très sévèrement critiqué quand il refuse d'éclairer le personnel de surveillance sur la pathologie dont souffre son patient. Le personnel pénitentiaire le soupçonne de mettre en danger sa sécurité en dissimulant des pathologies infectieuses ou en refusant de donner un diagnostic de pathologie mentale.
Dans un autre registre, le médecin peut être aussi rendu responsable de maintenir dans des lieux de détentions des patients justifiant ou semblant justifier, aux yeux du profane, de soins psychiatriques aigus alors qu'aucune clinique psychiatrique ne peut, ou ne veut accueillir de tels patients ...

Même si ces éléments semblent nous éloigner de la situation en cause, ils interviennent souvent lourdement dans l'appréciation clinique d'un cas comme celui dont il est question.

Tiraillé entre son devoir d'assistance et la pression de la sécurité, le médecin qui intervient en milieu pénitentiaire se devrait d'être solidement étayé par des règles décisionnelles ou des standards de pratiques. Pourtant, hormis les directives mentionnées plus haut, rien n'existe en la matière. Les autorités sanitaires semblent avoir souvent déserté cet espace de pratique qui concerne pourtant plusieurs milliers de personnes en situation de vulnérabilité. La création de services médicaux indépendants rattachés aux services de la santé publique est un moyen de donner étayage, soutien et indépendance à ces médecins de premier recours confrontés à de tels dilemmes. Pourtant cette recommandation, soutenue avec force par beaucoup, et figurant dans de nombreuses directives internationales, n'a que peu été suivie dans les faits.

En prison, comme ailleurs, le médecin doit faire preuve d'une clinique irréprochable dans des domaines variés (cliniques des addictions, psychiatrie, etc.), d'une grande rigueur diagnostique, mais aussi d'une connaissance particulière du milieu et des règles de droit. Il doit, en outre, user, en tout temps, d'une grande diplomatie avec ce milieu particulier en respectant les missions du personnel pénitentiaire qui souvent s'éloignent fort du soin. Il ne dispose souvent, il convient de le rappeler, que de moyens dérisoires, de locaux inadaptés et d'un temps trop restreint pour avoir la possibilité d'une appréciation approfondie.

\section{La solitude des médecins dans le cas présenté}

Dans cette douloureuse affaire, nos collègues ont fait la pénible expérience de la solitude: solitude face à une situation médicale particulière: Le patient se trouvait-il véritablement en dette hydrique? Le tableau présenté par le patient était-il le reflet d'une protestation caractérisée? Etait-il la manifestation clinique d'un trouble de la personnalité ou d'une autre pathologie? Comme tout médecin, ils ont apprécié l'urgence d'une si- 
tuation et pris les mesures qui leur apparaissaient les plus adéquates en fonction de leur observation et des indications qui leur étaient transmises.

Solitude aussi face aux experts mandatés, qu'ils estiment peu au fait des réalités de la médecine pénitentiaire et qui auraient privilégié une hypothèse quant aux causes du décès qui s'éloigne manifestement de la réalité clinique à laquelle ils ont eu affaire.

Une expertise médicale doit être menée avec la plus grande rigueur. L'expert doit être particulièrement attentif à ses limites de compétence et

\section{L'appréciation d'une urgence médicale en prison est toujours délicate}

éviter de se contenter d'apriori sur le fonctionnement d'une institution qu'il ne connaît pas. Le juge qui désigne l'expert doit aussi s'assurer que celui-ci dispose des compétences requises, non seulement dans la discipline médicale visée, mais aussi pour apprécier le contexte de l'exercice.

Cela amène à insister sur quelques évidences trop souvent oubliées. Une expertise doit obéir à des critères de qualité définis par des institutions reconnues (instituts de médecine légale, société de discipline, etc.). Mais une expertise doit aussi être capable de prendre en considération avec objectivité les paramètres mentionnés plus haut et les resituer dans un contexte aussi spécifique qu'une situation de tension générée par une grève de la faim en milieu pénitentiaire où le patient se manifeste par des troubles du comportement qui rendent difficile, voire faussent, l'évaluation clinique.

Une expertise doit enfin traduire en termes intelligibles pour le juge des appréciations médicales souvent complexes. Elle doit aussi permettre de comprendre comment une décision médicale qui a pour conséquence une prescription, un geste d'urgence ou un transfert en milieu hospitalier s'élabore en s'appuyant sur une observation, une élaboration diagnostique souvent malaisée et un raisonnement qui permet la pesée d'intérêts et la définition de priorités. Toutes ces démarches qui fondent l'acte médical sont souvent biaisées et complexifiées par le contexte carcéral.

\section{Beaucoup de progrès restent à faire dans la médecine pénitentiaire}

La Conférence des Médecins Pénitentiaires Suisses défend avec insistance la reconnaissance de la pratique particulière du médecin pénitentiaire, la nécessité de l'indépendance de cette pratique, le développement de standards de référence, etc.

Avec le soutien de la FMH, elle a initié une enquête sur la manière dont les directives de l'ASSM sont connues, et reconnues, par les autorités judiciaires et pénitentiaires, mais aussi par les médecins qui pratiquent dans ces institutions particulières. C'est un premier pas pour aider cette médecine particulière à disposer des références spécifiques qui font cruellement défaut, comme le soulignent nos collègues.

S'il est encore, dans notre pays, un domaine où beaucoup de progrès restent à faire, c'est bien la médecine pénitentiaire. Les autorités sanitaires et judiciaires doivent impérativement donner un statut et une reconnaissance aux soignants qui interviennent en prison pour que l'âpreté du milieu, la difficulté de la clinique médicale en milieu pénitentiaire et la spécificité de leur mission puissent enfin être prises en compte, autant par les pouvoirs publics que par l'université. Il est fondamental, en effet, que les autorités reconnaissent que les médecins pénitentiaires doivent bénéficier d'un statut qui leur garantit des conditions de pratique décentes et une nécessaire indépendance. Il est aussi de l'intérêt de tous que la médecine pénitentiaire puisse devenir un objet d'enseignement et de recherches reconnu par les facultés de médecine. 